\title{
Mechanical model of a pre-stressed piezoelectric buckling actuator
}

\author{
Florian Toth ${ }^{1}$, Manuel Dorfmeister ${ }^{2}$, Manfred Kaltenbacher ${ }^{1}$ \\ ${ }^{1}$ Institute of Mechanics and Mechatronics, TU Wien, Vienna, Austria \\ 2 Institute of Sensor and Actuator Systems, TU Wien, Vienna, Austria \\ florian.toth@tuiwen.ac.at
}

\begin{abstract}
:
A mechanical model for a piezoelectric plate actuator is developed. The effects of the piezo-actuation as well as the equivalent effect of thermal pre-stress are included in the constitutive model. Based on classical lamination theory we derive a formulation for the plate behavior including piezoelectric effects and thermal strains. Using von Karman plate theory we obtain equilibrium equations able to describe the non-linear behavior of the plate. The equilibrium equations are numerically solved for axisymmetric plates using the shooting method to obtain equilibrium paths.
\end{abstract}

Key words: MEMS, actuator, buckling, piezo, plate

\section{Introduction}

Loss of stability through buckling or snapthrough is usually undesired in engineering structures. However, buckling phenomena can also be exploited e.g. to determine material parameters through indirect experiments [1], or in actuator applications [2]. While the determination of critical loads (i.e. the load at which a bifurcation occurs) is often sufficient for the prevention against the loss of stability in standard applications, the exploitation of buckling phenomena in actuator applications requires precise knowledge about the postcritical behavior of the investigated system.

The investigated microelectromechanical system (MEMS) consists of a circular, multilayer plate with the intended use as an actuator for digital sound reconstruction (DSR). The multilayer structure is pre-stressed due to the production process leading to buckling of the plate structure. The piezo layer is actuated to switch from one to the other stable equilibrium. The advantage of such an actuator exploiting the post-buckling behavior is a significantly larger stroke level.

\section{Mechanical Model}

One of the key points of the mechanical modelling of the actuator system is the correct description of the electromechanical coupling including pre-stress in a large-displacement framework. However, the model must be simple enough to enable a mathematical analysis of the non-linear behavior of the structure to be suitable for the design of actuators. Therefore, some simplifications are employed: As we are dealing with thin plate-like structures, we assume a plane stress state with the axis of the vanishing normal stress oriented perpendicularly to the plane of the plate. Adopting the Kirchhoff hypothesis we can describe the strain state in the plate by

$\bar{\epsilon}=\epsilon+z \kappa$

where $z$ denotes the transverse coordinate measured from the plate's reference surface. The deformed shape of the reference surface is described by the in-plane strain tensor $\varepsilon$ and the curvature tensor $\kappa$. In order to account for large displacements we use the strain measures introduced by von Karman

$$
\begin{aligned}
& \epsilon=\frac{1}{2}\left(\nabla u+(\nabla u)^{T}+\nabla u_{3} \nabla u_{3}\right) \\
& \kappa=-\nabla \nabla u_{3}
\end{aligned}
$$

which allow to model the coupling of membrane strains, and the transverse displacements, i.e. the third component of the displacement vector of the reference surface $u=\left[u_{1}, u_{2}, u_{3}\right]$.

All materials are modelled as transversally isotropic with the axis of the vanishing normal stress perpendicular to the isotropic plane. Employing Voigt's linearized theory of piezoelectricity and additionally considering thermal strains we can write the linearized constitutive relation as 


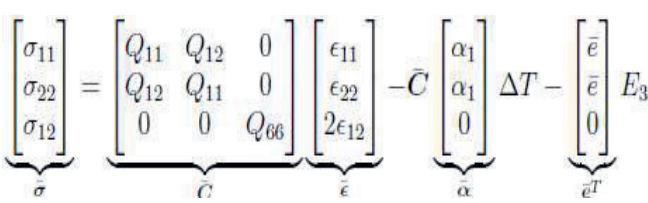

and

$D_{3}=\bar{e} \bar{\epsilon}-\bar{e} \bar{\alpha} \Delta T+\bar{\eta} E_{3}$

where the entries in the effective plane stress stiffness tensor (written in Voigt notation) are computed as

$Q_{11}=C_{11}-\frac{C_{13}^{2}}{C_{33}}, \quad Q_{12}=C_{12}-\frac{C_{13}^{2}}{C_{33}}, \quad Q_{66}=\frac{C_{11}-C_{12}}{2}$, from the usual $C_{i j}$, which denote the non-zero entries of the transversally isotopic stiffness tensor with 1-2 as the isotropic plane. The entries of the effective plane stress piezoelectric coupling tensor compute as

$\bar{e}=e_{31}-\frac{C_{13}}{C_{33}} e_{33}$,

and the effective electric permittivity becomes

$\bar{\eta}=\eta_{33}+\frac{e_{33}^{2}}{Q_{33}}$

A key point in the mechanical description of thin piezoelectric structures is the assumption that Gauss' law reduces to a one-dimensional form if we can neglect the in-plane components. Thus, we have

$\partial D_{3} / \partial z=0$

meaning that the dielectric displacement is constant in each layer. Integrating eq.(5) over the layer thickness we obtain

$D_{3}=\frac{1}{\Delta z_{l}} \int_{z_{l}}^{z_{l+1}} D_{3} \mathrm{~d} z=\overline{\boldsymbol{e}} \boldsymbol{\epsilon}+\bar{z} \overline{\boldsymbol{e}} \boldsymbol{\kappa}-\overline{\boldsymbol{e}} \overline{\boldsymbol{\alpha}} \Delta T+\bar{\eta} \frac{\Delta V}{\Delta z}$

which we can insert back into eq. (5) to obtain an expression for the electric field strength in transverse direction

$$
E_{3}=\frac{\bar{z}-z}{\bar{\eta}} \bar{e} \kappa+\frac{\Delta V}{\Delta z} \text {. }
$$

We can now use this relation, eq. (7), and insert it in eq. (4) to obtain the final constitutive relation

$\sigma=\bar{C} \epsilon+z(\bar{C}-(\bar{z}-z) \bar{E}) \kappa-\tau$

which describes the behavior of each piezoelectric, thermally stressed layer. We note that the tensor

$\bar{E}=\frac{\bar{e}^{T} \bar{e}}{\bar{\eta}}$ describes the additional bending stiffness contribution due to piezoelectricity. Another important observation is that pre-stress, introduced here via a thermal strain acts exactly like an actuated piezo layer. Thus, both effects can be include via a single term

$\tau=\bar{C} \bar{\alpha} \Delta T+\bar{e}^{T} \frac{\Delta V}{\Delta z}$

As it is clear that both effects, piezo actuation and pre-stress enter the equation in the same way by the effective in-plane stress $T$ we will not consider them separately.

We now obtain the stresses over the thickness and sum over the individual layer to obtain the section forces and moments

$n=\sum_{l} \int \bar{\sigma}_{l} \mathrm{~d} z$ and $m=\sum_{l} \int z \bar{\sigma}_{l} \mathrm{~d} z$ respectively. The constitutive law on the plate level can then be written as

$\left[\begin{array}{c}n \\ m\end{array}\right]=\left[\begin{array}{ll}A & B \\ B & D\end{array}\right]\left[\begin{array}{l}\epsilon \\ \kappa\end{array}\right]-\left[\begin{array}{c}\tilde{n} \\ \tilde{m}\end{array}\right]$

The components of above equation are

$\tilde{n}=\sum_{l} \int \tau_{l} \mathrm{~d} z \quad \tilde{m}=\sum_{l} \int z \tau_{l} \mathrm{~d} z$

$A=\sum_{l} \Delta z_{l} \bar{C}_{l} \quad B=\sum_{l} \Delta z_{l} \bar{C}_{l}$

$D=\sum_{l} \int\left(z^{2}\left(\overline{\boldsymbol{C}}_{l}+\overline{\boldsymbol{E}}_{l}\right)-z \bar{z}_{l} \overline{\boldsymbol{E}}_{l}\right) \mathrm{d} z$

where we have introduced the layer thickness and the middle position of a layer

$\Delta z_{l}=z_{l+1}-z_{l} \quad$ and $\quad \bar{z}_{l}=\frac{z_{l+1}+z_{l}}{2}$.

Finally, we write the equilibrium equations for the plate as

$\nabla \cdot n=0$

$\boldsymbol{\nabla} \cdot \boldsymbol{\nabla} \cdot \boldsymbol{m}+\boldsymbol{\nabla} \cdot\left(\boldsymbol{n} \cdot \boldsymbol{\nabla} u_{3}\right)-p_{3}=0$

where $p_{3}$ denotes a pressure force acting in transverse direction. We have neglected inplane forces in eq. (12) and are only interested in the static equilibrium. Note that all relations have been written in coordinate-free form, i.e. they are applicable both to e.g. Cartesian and Cylindrical coordinates.

\section{Numerical Solution}

As we are considering circular plates we apply the divergence and gradient operators in eqs. 
(12) and (13) in cylindrical coordinates. If we restrict ourselves to circular symmetric solutions, we obtain two coupled, non-linear ordinary differential equations (ODEs). The ODEs are fourth-order in $\mathrm{u}_{3}$, the transverse direction, and third order in $u_{r}$, the radial direction. The equation for the circumferential direction is trivially satisfied. By simply introducing additional variables, we can write the equations as a non-linear system of 7 first order ODEs.

We can solve the boundary value problem numerically by the shooting method. Although, this is in principle a simple procedure one needs to consider some details: Firstly the integration must be started at a small distance to $r=0$ due to the singularity of the equations. Secondly, the chosen start value should satisfy the boundary conditions [7]. Finally, one needs to iteratively find the correct start values such that the boundary conditions at the outer edge are satisfied. This is done using the NelderMead algorithm [5]. For the forward integration we employ a $4^{\text {th }}$ order Runge-Kutta scheme [6].

\section{Results}

We compute equilibrium paths for clamed plates under different in-plane loadings and transverse pressures. For the sake of simplicity, and to test the implementation we consider isotropic plates with a single layer. Introducing a transverse pressure acts as an imperfection similar to the one introduced by an unsymmetrical layup. However, we do not have any coupling between membrane loading and curvature change, i.e. the B-matrix is zero.

Results were obtained for a plate with thickness to radius ratio of 0.01 . The applied in-plane loads were normalized by the product of the inplane stiffness the square of the thickness to radius ratio and the thickness. The transverse pressure was normalized by the product of inplane stiffness and the fifth power of the thickness to radius ratio.

The equilibrium obtained curves are depicted in Fig. 1. Following the dashed line for the lowest pressure we are very close to the trivial

\section{References}

[1] E.A. Wilder, S. Guo, S. Lin-Gibson, M.J. Fasolka, C.M. Stafford, Macromolecules, American Chemical Society (ACS), 39, 4138-4143 (2006); doi: $10.1021 / \mathrm{ma} 060266 \mathrm{~b}$

[2] D. Yang, B. Mosadegh, A. Ainla, B. Lee, F. Khashai, Z. Suo, K. Bertoldi, G.M. Whitesides, Advanced Materials, Wiley-Blackwell, 27, 63236327 (2015); doi: 10.1002/adma.201503188 equilibrium path, until the solution bifurcates at the first buckling load of 1.22 [4]. Choosing a suitable starting value one can also follow overcritical equilibrium paths which will again bifurcate at the second buckling load. Considering the solutions for higher transverse pressure we observe multiple possible equilibrium positions for overcritical loads.

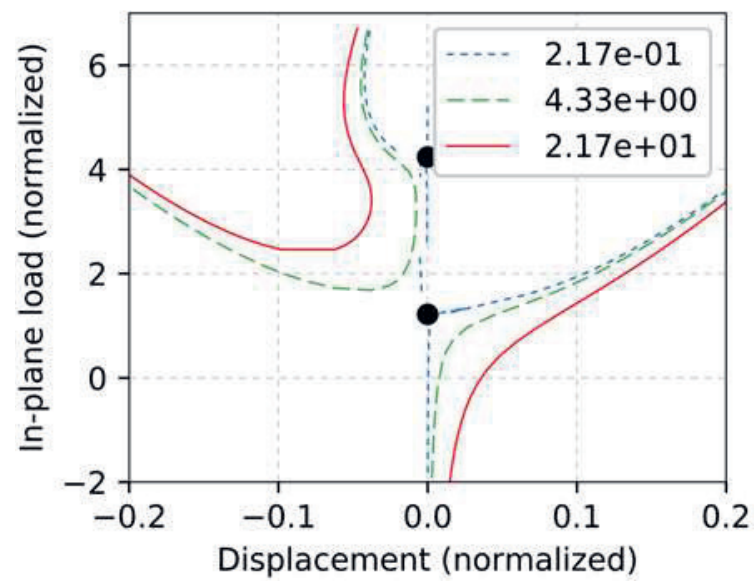

Fig. 1. Load displacement diagram for transverse center displacement versus in-plane load for different values of the transverse pressure. The theoretical buckling loads are indicated as black dots.

It should be noted that the present method, while very useful to obtain equilibrium paths, does not allow determining the stability of the equilibrium. Additionally, the assumption of axial symmetry does not allow the description of snap-buckling, i.e. the transfer from one overcritical equilibrium path to another by an unsymmetrical buckling mode [3].

\section{Summary}

A formulation for piezoelectric plate actuators has been presented. The mathematically equivalent effects of thermal pre-strain and piezo actuation are included into the formulation by a modified constitutive law. They both can be described by an equivalent in-plane stress. The equilibrium equations based on the von Karman plate theory were solved numerically for circular plates with symmetric deformation figure by the shooting method

[3] M. Krommer, Y. Vetyukov, E. Staudigl, Smart Structures and Systems, Techno-Press, 2016, 18, 155-181; doi: 10.12989/sss.2016.18.1.155M.

[4] W.C. Young, R.J. Roark, R.G. Budynas, McGraw-Hill, 2002

[5] J.A. Nelder, R.A. Mead, The Computer Journal, $1965,7,308-13$

[6] E. Hairer, S.P. Norsett and G. Wanner, Springer Series in Computational Mathematics, SpringerVerlag (1993) 
[7] Y.-G. Wang, J.-L. Shi, X.-Z. Wang, Applied Mathematical Modelling, Elsevier BV, 2009, 33, 3523-3532; doi: 10.1016/j.apm.2008.11.016 